\title{
Isolated Intraspinal Extradural Tubercular Lesion without Osseous Involvement
}

\author{
${ }^{1}$ Department of Neuro Surgery, PGIMER, Dr. Ram Manohar Lohia \\ Hospital, New Delhi, India \\ 2Department of Clinical Microbiology, Northern Railway Central \\ Hospital, New Delhi, India \\ ${ }^{3}$ Department of Neuro Surgery, Sir Sunder Lal Hospital, IMS, \\ Banaras Hindu University, Varanasi, Uttar Pradesh, India \\ ${ }^{4}$ Department of Pathology, Sir Sunder Lal Hospital, IMS, \\ Banaras Hindu University, Varanasi, Uttar Pradesh, India \\ Indian J Neurosurg 2018;7:272-274
}

Sharad Pandey ${ }^{1}$ Deepa Pandey ${ }^{2} \quad$ Kulwant Singh ${ }^{3} \quad$ Vivek Sharma $^{3} \quad$ Amrita Ghosh $^{4}$

Address for correspondence Sharad Pandey, MS, MCh, Department of Neuro Surgery, PGIMER, Dr. Ram Manohar Lohia Hospital, New Delhi 110001, India (e-mail: drsharad23@yahoo.com).

\begin{abstract}
Tuberculosis (TB) continues to be a major public health problem in developing countries. Approximately $10 \%$ of patients with extrapulmonary TB have skeletal involvement. The spine is the most commonly affected skeletal site. The neurologic manifestations of spinal

Keywords

- tuberculosis

- intraspinal extradural lesion

- without osseous involvement TB are most often secondary to bone involvement but can also be due to neural and perineural tissues involvement. TB may involve any level from the cervical to lumbosacral region. Extradural tubercular granuloma without vertebral involvement is uncommon. The authors reported an uncommon case of isolated intraspinal extradural TB lesion without osseous involvement of the thoracic spine in an adult man. Surgical intervention along with chemotherapy is one of the preferred treatments for intraspinal extradural tubercular lesion.
\end{abstract}

\section{Introduction}

Spinal tuberculosis (TB) comprises nearly $50 \%$ of the skeletal TB. ${ }^{1,2}$ Most frequently involved are the upper lumbar and lower thoracic spine. Spinal TB is characterized by destruction of the intervertebral disc space and adjacent vertebral bodies, collapse of the spinal elements, and anterior wedging leading to the characteristic angulation and gibbus (palpable deformity due to involvement of multiple vertebrae) formation. The neurologic manifestations of spinal TB are most often secondary to bone involvement but can also be due to neural and perineural tissues involvement, that is, epidural space, subdural space, meninges, or cord tissue directly. Intraspinal extradural tubercular lesion is a rare cause of compressive spinal cord lesion, and especially infrequent is an isolated granuloma without vertebral involvement. ${ }^{3,4}$ Interestingly, no study has explained the reasons for this localized involvement. We reported an uncommon case of isolated intraspinal extradural tuberculous lesion without osseous involvement of the thoracic spine.

\section{Case Report}

A 21-year-old adult man presented at the neurosurgery OPD with complaints of backache in the mid-thoracic region since

received

April 18, 2016

accepted

June 20, 2016

published online

November 27, 2018
1 to 2 months and progressively increasing weakness in both the lower limbs since 15 to 20 days. There was no history of trauma, fever, chronic cough, weight loss, and antitubercular treatment (ATT). No bowel and bladder complaints were present. No significant family history of Koch's was present. On physical examination, slight tenderness was present in the upper thoracic region and sensory loss was found below D6 spinal level. Power in both the lower extremities was 2/5 with normal muscle tone. Deep tendon reflexes (DTRs) in both the lower limbs were hyperactive. Babinski's sign was positive. Routine laboratory examination was within normal limits, except erythrocyte sedimentation rate (ESR) that was $25 \mathrm{~mm} / \mathrm{h}$. Conventional radiograph of the chest and dorsolumbar spine were normal, without any evidence of vertebrae destruction. Magnetic resonance imaging (MRI) of the spine showed relatively well-defined $\mathrm{T} 1$ isointense intraspinal extradural mass lesion measuring $4 \mathrm{~cm} \times 3 \mathrm{~cm}$ at D6 vertebral level, causing mass effect on adjacent thoracic spinal cord evident by anterior displacement and compression without obvious cord signal intensity changes. Minimal postcontrast enhancement of mass was seen in T1 contrast (-Fig. 1). No extension into bilateral neural foramina or bony involvement, no obvious sign of arachnoiditis, or bone
C2018 Neurological Surgeons' Society of India
License terms

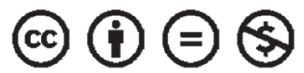

$10.1055 / \mathrm{s}-0038-1671793$ ISSN 2277-954X. 
marrow altered signal changes were noticed. Mid-saggital T2 images showed mildly hyperintense mass lesion posterior to the spinal cord at D6 level causing mass effect on cord. MRI features were suggestive of possibility of either nerve sheath tumor or inflammatory granuloma.

The patient underwent D5-D6 laminectomy and excision of lesion. Intraoperatively, posterior elements of the dorsal spine were intact, and the mass was totally extradural, adhered to the spinal dura mater. Lesion was gently separated from the dura mater and excised ( - Fig. 2). Lesion size was around $4 \mathrm{~cm} \times 2 \mathrm{~cm}$ and sent for histopathologic examination and microbiological culture. The cultures of the biopsy specimen were negative for anaerobes, fungi, and mycobacteria. Histopathologic investigation revealed chronic inflammation

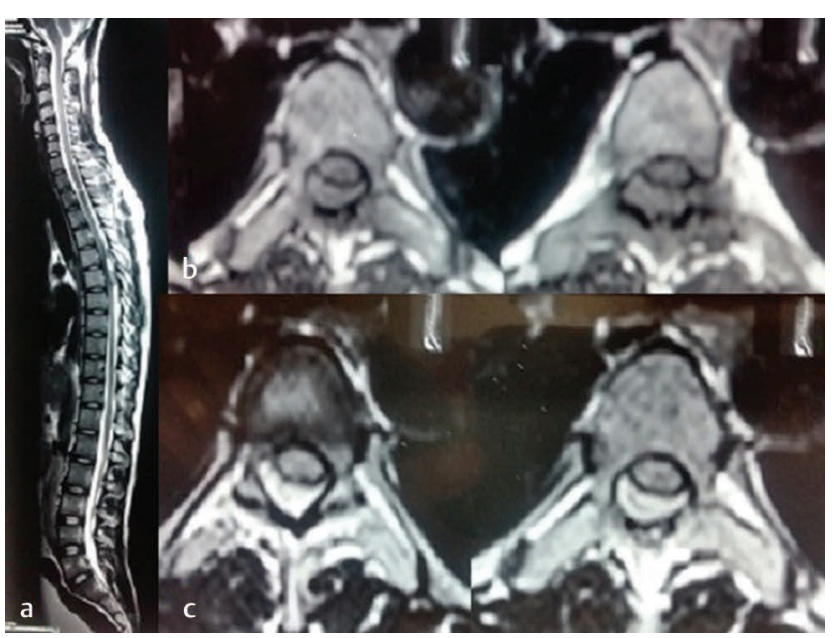

Fig. 1 (a) Magnetic resonance imaging of spine showing mid-saggital T2 images showing mildly hyper intense mass lesion posterior to spinal cord at D6 level causing mass effect on cord. (b) Axial section with T1 isointense intraspinal extradural mass lesion at D6 vertebral level. (c) Axial section with postcontrast enhancement of lesion in T1 contrast. and ill-formed granulomas embedded on a fibrocollagenous tissue (-Fig. 3). Great sheets of delicate vessels lined by a flattened endothelium and separated by scant connective tissue stroma were also observed. The patient was continued on ATT empirically. Neurologic symptoms improved, and he was discharged with moderate neurologic deficit. On follow-up, he showed remarkable improvement.

\section{Discussion}

Tuberculosis continues to be a major public health problem in developing countries despite widespread national TB programmes. Approximately $10 \%$ of patients with extrapulmonary ТB have skeletal involvement. The spine is the most commonly affected skeletal site, followed by the hip and knee. ${ }^{5}$ TB of the spine comprises approximately 25 to $60 \%$ of all osseous infections caused by TB., ${ }^{6,7}$

Owing to nonspecific history and widely varying spectrum of clinical features, its diagnosis needs special attention. Fever and systemic symptoms may not become evident until the late stage. Almost one-half of the patients with

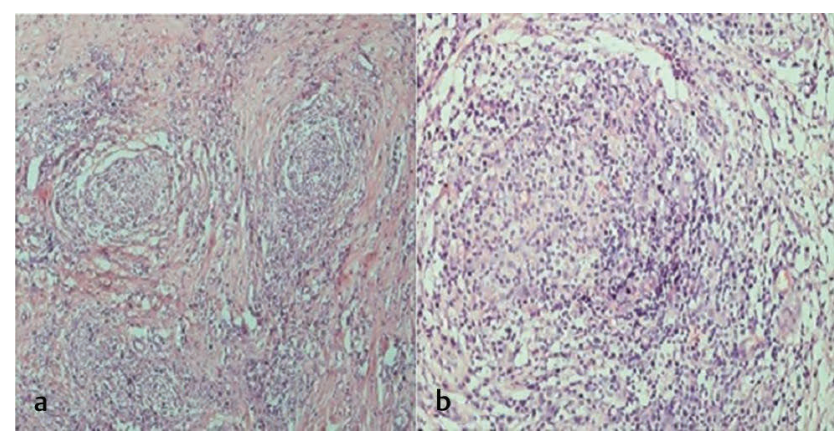

Fig. 3 (a) Microphotograph showing scattered ill-formed granulomas embedded on a fibrocollagenous tissue (H\&E, 100X). (b) Microphotograph showing high-power view of ill-formed granuloma (H\&E, 400X).

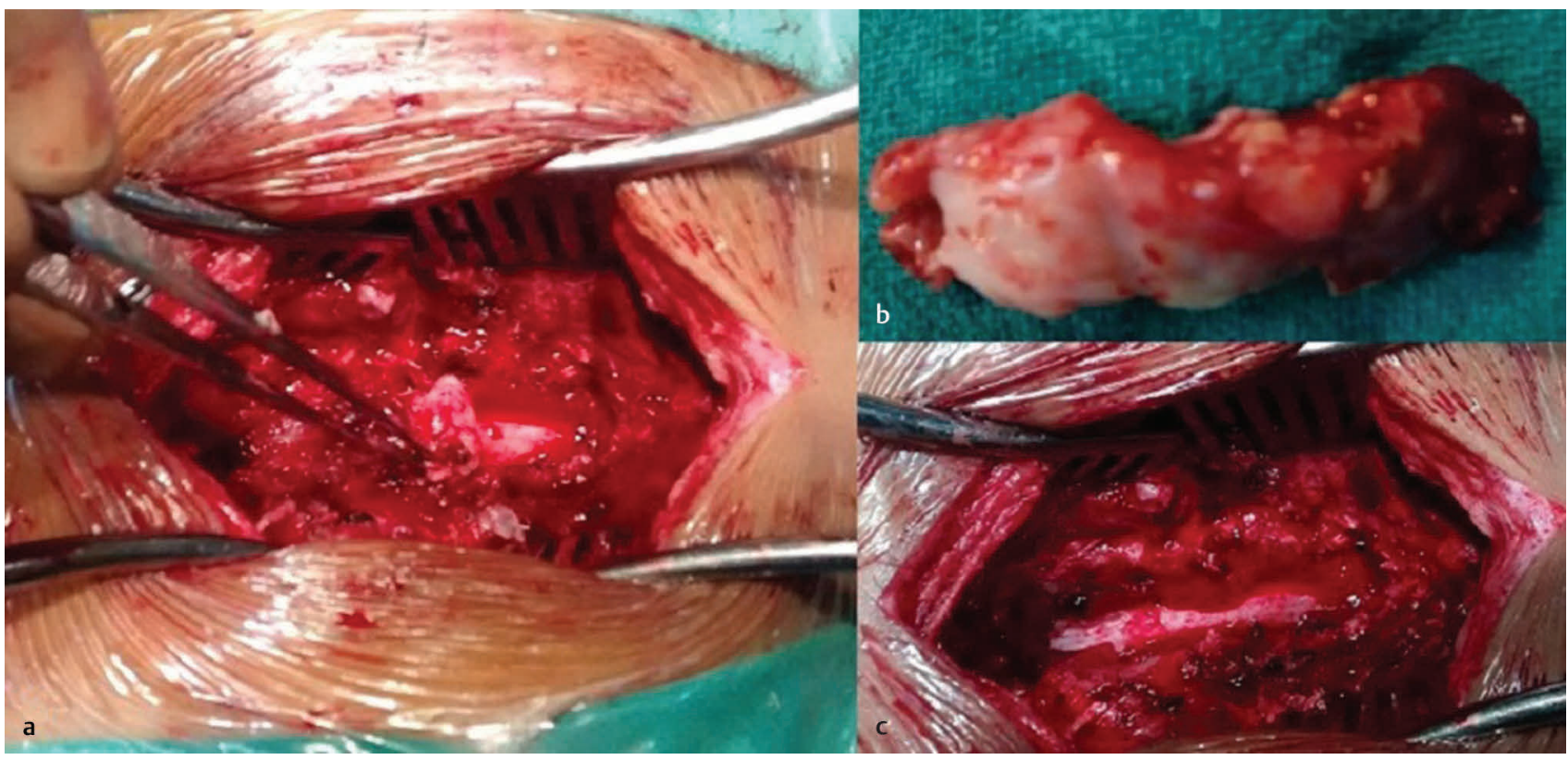

Fig. 2 (a) Lesion after D5-D6 laminectomy showing extradural lesion adhered to the spinal dura mater. (b) Excised lesion. (c) Whitish spinal dura mater after lesion excision. 
extrapulmonary TB have normal chest X-ray findings. ${ }^{8}$ ESR, though nonspecific, is found to be elevated in most cases.

Tuberculosis may involve any level from the cervical to lumbosacral region. ${ }^{9}$ Extradural tubercular granuloma without vertebral involvement is uncommon. Also, isolated intraspinal TB without radiologic evidence of vertebral involvement is rather uncommon. Kumar et al reported 22 cases with intraspinal TB, in which 3 out of 12 cases with extradural granulomas were without osseous involvement. ${ }^{10}$ We reported another such similar case of tubercular granuloma in an adult male without vertebral involvement of the thoracic region.

Among spinal TB, isolated intraspinal TB with absence of vertebral destruction and with neural deficit is less than $5 \%$.

Multiple imaging modalities such as conventional radiography, scintigraphy, computed tomography (CT), and myelography have all been reported to be helpful in the diagnosis of spinal TB, but MRI is relatively more sensitive and is believed to be the modality of choice. ${ }^{11,12}$

The final diagnosis is established by histologic examination. It is suggested that tubercular granuloma should be considered in the differential diagnosis when a case of spinal tumor syndrome is encountered in an endemic area of TB. Without losing the precious time, ATT should be started when the investigations are awaited to avoid treatment delay and the patient be monitored timely.

As per the "Standards for TB Care in India" guidelines by the World Health Organization (WHO), all new patients should receive an internationally accepted first-line treatment regimen. The initial phase consists of treatment with isoniazid $(\mathrm{H})$, rifampicin $(\mathrm{R})$, pyrazinamide $(\mathrm{Z})$, and ethambutol (E) for 2 months followed by the continuation phase comprising three drugs $\mathrm{H}, \mathrm{R}, \mathrm{E}$ given for at least 4 months. Owing to risk of disability and mortality and difficulties in assessing the treatment response, WHO recommends that the duration of continuation phase may be extended by 3 to 6 months in special situations such as bone and joint TB, spinal TB with neurologic involvement, and neurotuberculosis. ${ }^{13}$

Surgical intervention along with ATT is one of the preferred treatments for intraspinal extradural tubercular lesion. Combination of microsurgical resection and antitubercular chemotherapy provides promising results. Similar were the suggestions by Türeyen regarding optimal treatment for isolated intraspinal extradural tubercular lesion. ${ }^{14}$ With this treatment plan, patients usually show good neurologic recovery after adequate surgical decompression and necessary antitubercular treatment. In this case also, the patient showed good neurologic recovery with this treatment modality.

Although isolated intraspinal extradural tubercular lesion without osseous involvement is an uncommon entity, but it should be kept in mind as a possible differential diagnosis in cases of extradural spinal lesions.

\section{Conflict of Interest}

None.

\section{References}

1 Hsu LCS, Leong JCY. Tuberculosis of the lower cervical spine (C2 to C7). A report on 40 cases.J Bone Joint Surg Br 1984;66(1):1-5

2 Gautam MP, Karki P, Rijal S, Singh R. Pott's spine and paraplegia. JNMA J Nepal Med Assoc 2005;44(159):106-115

3 Mantzoros CS, Brown PD, Dembry L. Extraosseous epidural tuberculoma: case report and review. Clin Infect Dis 1993; 17(6):1032-1036

4 Reichenthal E, Cohen ML, Shalit MN. Extraosseous extradural tuberculous granuloma of the cervical spine: a case report and review of intraspinal granulomatous infections. Surg Neurol 1981;15(3):178-181

5 Garg RK, Somvanshi DS. Spinal tuberculosis: a review. J Spinal Cord Med 2011;34(5):440-454

6 Harada Y, Tokuda O, Matsunaga N. Magnetic resonance imaging characteristics of tuberculous spondylitis vs. pyogenic spondylitis. Clin Imaging 2008;32(4):303-309

7 Tali ET. Spinal infections. Eur J Radiol 2004;50(2):120-133

8 Simon HB. Infections due to mycobacteria. In: Rubinstein NE, Federman D, eds. Scientific American Medicine Update 6/94. Vol. 1. New York, NY: Scientific American Medicine; 1994

9 Ohaegbulam SC. Spinal extraosseous extradural tuberculoma. Tubercle 1977;58(2):97-99

10 Kumar S, Jain AK, Dhammi IK, Aggarwal A. Treatment of intraspinal tuberculoma. Clin Orthop Relat R 2007:460:62-66

11 Shanley DJ. Tuberculosis of the spine: imaging features. AJR Am J Roentgenol 1995;164(3):659-664

12 Sharif HS, Clark DC, Aabed MY, et al. Granulomatous spinal infections: MR imaging. Radiology 1990;177(1):101-107

13 Standard for TB care in India. Central TB Division, Ministry of Health and Family Welfare, Government of India and WHO 2014. http:www.tbcindia.nic.in

14 Türeyen K. Tuberculoma of the conus medullaris: case report. Neurosurgery 2002;50(3):651-652, discussion 652-653 